\title{
Truncated star formation in dwarf spheroidal galaxies and photometric scaling relations
}

\author{
Sven De Rijcke ${ }^{1}$, Sander Valcke ${ }^{1}$, Christopher J. Conselice ${ }^{2}$, \\ and Samantha Penny ${ }^{2}$ \\ ${ }^{1}$ Sterrenkundig Observatorium, University of Ghent, \\ Krijgslaan 281, S9, B-9000 Gent, Belgium \\ email: sven.derijcke@UGent.be, sander.valcke@UGent.be \\ ${ }^{2}$ School of Physics and Astronomy, University of Nottingham, University Park, NG9 2RD, UK \\ email: conselice@nottingham.ac.uk, ppxsp@nottingham.ac.uk
}

\begin{abstract}
We investigate the global photometric scaling relations traced by early-type galaxies in different environments, ranging from dwarf spheroidals, over dwarf elliptical galaxies, up to giant ellipticals $\left(-8 \mathrm{mag} \gtrsim \mathrm{M}_{V} \gtrsim-24 \mathrm{mag}\right.$ ). These results are based in part on our new HST/ACS F555W and F814W imagery of dwarf spheroidal galaxies in the Perseus Cluster. We show that at $M_{V} \sim-14 \mathrm{mag}$, the slopes of the photometric scaling relations involving the Sérsic parameters change significantly. We argue that these changes in slope reflect the different physical processes that dominate the evolution of early-type galaxies in different mass regimes. We present N-body/SPH simulations of the formation and evolution of dwarf spheroidals that reproduce these slope changes and discuss the underlying physics. As such, these scaling relations contain a wealth of information that can be used to test models for the formation of early-type galaxies.
\end{abstract}

Keywords. galaxies: dwarf, galaxies: photometry, galaxies: structure

\section{Introduction}

Bright elliptical galaxies, or Es, and dwarf elliptical galaxies, or dEs, follow the same photometric and kinematic scaling relations (Graham \& Guzmán 2003, Matković \& Guzmán 2005, De Rijcke et al. 2005, Smith Castelli et al. 2008). In the luminosity interval -24 mag $\lesssim M_{V} \lesssim-14$ mag the parameters of the Sérsic profile follow simple power-laws as a function of luminosity and early and late type galaxies trace parallel Tully-Fisher relations (De Rijcke et al. 2007). From this wealth of data a picture of (dwarf) galaxy formation emerges that suggests an underlying unity in the physics driving the formation and evolution of stellar systems, with the environment playing a role that is in many situations subordinate to that of internal processes. More specifically, numerical simulations and semi-analytic models of galaxy formation within a $\Lambda$ CDM cosmology can account for the observed scaling relations when taking into account supernova feedback in galactic gravitational potential wells steepening with galaxy mass (Carraro et al. 2001, Nagashima \& Yoshii 2004, Ricotti \& Gnedin 2005, Marcolini et al. 2006, Valcke, De Rijcke, Dejonghe 2008).

In this contribution, we investigate whether the photometric scaling relations traced by dEs and Es persist down to the dSphs $\left(\mathrm{M}_{V} \gtrsim-14 \mathrm{mag}\right)$. 


\section{Photometric data}

\subsection{Perseus Cluster data}

We have obtained high resolution Hubble Space Telescope (HST) Advanced Camera for Surveys (ACS) WFC imaging in the F555W and F814W bands of five fields in the Perseus Cluster core, in the immediate vicinity of NGC1275 and NGC1272, the cluster's brightest members, obtained in 2005 (program GO 10201). The scale of the images is $0.05^{\prime \prime}$ pixel $^{-1}$, with a field of view of $202^{\prime \prime} \times 202^{\prime \prime}$, providing a total survey area of $\sim 57 \mathrm{arcmin}^{2}$. Exposure times were 2368 and 2260 seconds for the F555W and F814W bands, respectively. The fields were chosen to cover the most likely cluster dSphs and dEs identified from ground-based imagery by Conselice, Gallagher, Wyse (2003). For some of these, there is spectroscopic confirmation of their cluster membership (Penny \& Conselice 2008). For the others, we use morphological criteria to decide cluster membership. The CAS system for quantifying compactness, asymmetry, and clumpiness/smoothness (Conselice 2003b) proves very useful for rejecting e.g. background spiral galaxies based on a smoothness criterion and background bright ellipticals based on a compactness criterion (see Penny et al. 2008). The Perseus dataset straddles the dE-dSph transition at $\mathrm{M}_{V} \sim-14 \mathrm{mag}$ and is therefore essential to the discussion that follows.

\subsection{Data from the literature}

The photometric data, including resolved photometry for surface brightness profiles, of the Local Group dSphs that are identified as Milky Way satellites are collected from Grebel, Gallagher, Harbeck (2003) and Irwin \& Hatzidimitriou (1995), adopting the distances listed in Grebel, Gallagher, Harbeck (2003). Data of the M31 dSph satellites is taken from Peletier (1993), Caldwell (1999), Grebel, Gallagher, Harbeck (2003), McConnachie \& Irwin (2006), McConnachie, Arimoto, Irwin (2007), and Zucker et al. (2007). Data of three Local Group dSphs that are not linked to a giant host galaxy, the Tucana dSph, DDO210, and KKR25, come from Saviane, Held, Piotto (1996), Grebel, Gallagher, Harbeck (2003), and McConnachie \& Irwin (2006). De Rijcke et al. (2005) (D05) and Mieske et al. (2007) provide photometric data on the early-type dwarf galaxy population of the Fornax cluster. Half of the D05 sample consists of dEs from the NGC5044 and NGC5989 groups. The data of the dSphs and dEs in the Antlia cluster are taken from Smith Castelli et al. (2008). Data for the giant elliptical and for Coma dEs is taken from Graham \& Guzmán (2003) (GG03).

This sample of early-type galaxies comprises dwarf spheroidals, with -14 mag $\lesssim \mathrm{M}_{V} \lesssim$ $-8 \mathrm{mag}$, dwarf ellipticals, with $-19 \mathrm{mag} \lesssim \mathrm{M}_{V} \lesssim-14 \mathrm{mag}$, and bright ellipticals, with $\mathrm{M}_{V} \lesssim-19 \mathrm{mag}$. We plot the positions of the sample galaxies in diagrams of $\mathrm{V}$-band absolute magnitude vs. (i) half-light radius $\mathrm{R}_{\mathrm{e}}$ (in $\mathrm{kpc}$ ), vs. (ii) the Sérsic exponent $n$ of the best fitting Sérsic profile, vs. (iii) the central V-band surface brightness of the best fitting Sérsic profile, and vs. (iv) V-I colour.

For the Local Group dSphs for which no Sérsic parameters can be found in the literature, we fit Sérsic profiles, with an added constant background density of stars, to the star counts of the dSphs presented in Irwin \& Hatzidimitriou (1995).

We now place these early-type galaxies in diagrams correlating the $\mathrm{V}$-band absolute magnitude $\mathrm{M}_{V}$, the Sérsic exponent $n$, the extrapolated central surface brightness $\mu_{0, V}$, and the $\mathrm{V}-\mathrm{I}$ colour. The goal is to investigate the behaviour of the relations between these structural parameters as a function of luminosity in the range $-24 \mathrm{mag}<\mathrm{M}_{V}<-8 \mathrm{mag}$ and of environment, using galaxies from the Local Group; the NGC5044 and NGC5989 groups; and the Fornax, Perseus, and Coma clusters. 


\section{Photometric scaling relations}

For our Perseus dSphs/dEs, we measure the profiles of surface-brightness, position angle, and ellipticity as a function of the geometric mean of major and minor axis distance using our own software. Residual cosmics, background galaxies, and foreground stars are masked and not used in the fit. The shape of an isophote, relative to the best fitting ellipse, is quantified by expanding the surface brightness variation along this ellipse in a fourth order Fourier series. Apparent ABMAG magnitudes in the F555W and F814W bands are calculated using the zero-points given by Sirianni et al. (2005). These magnitudes are corrected for interstellar reddening adopting the color excess $\mathrm{E}(\mathrm{B}-\mathrm{V})=0.171 \mathrm{mag}$ (Schlegel, Finkbeiner, Davis 1998) and using the prescriptions given in Sirianni et al. (2005). These reddening-corrected magnitudes are finally converted into Johnson V and I band magnitudes using the transformations of Sirianni et al. (2005).

The smooth 2D surface brightness distribution is integrated over circular apertures out to the last isophote we could reliably measure (which is at $\mu_{\mathrm{ABMAG}} \approx 27 \mathrm{mag} \mathrm{arcsec}^{-2}$ in both the F555W and F814W images) to derive model independent structural parameters, such as the total apparent magnitude and the half-light radius in each band. For such deep images of galaxies with a roughly exponentially declining surface brightness profile, this truncation results in an insignificant uncertainty on the total luminosity, of the order of a few per cent (see also De Rijcke et al. 2005). V-I colors are measured using the V and I-band flux inside the I-band half-light radius. We fit a Sérsic profile to the V-band surface brightness profiles of the program galaxies, expressed in mag $\operatorname{arcsec}^{-2}$.

\section{Numerical simulations}

The basic SPH scheme of HYDRA was modied to include star formation, supernova feedback, chemical enrichment and gas cooling. The simulations start from a homogeneous gas cloud collapsing onto a Dark Matter (DM) halo. Based on a suite of numerical

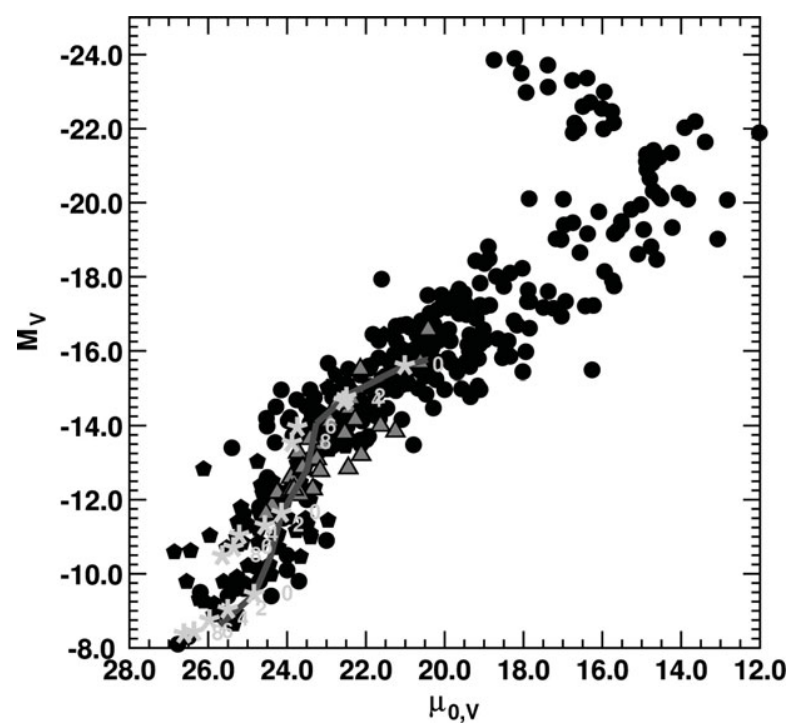

Figure 1. Absolute V-band magnitude versus Sérsic central surface brightness. The dSphs deviate significantly from the dEs and Es (black data points). The models of Valcke, De Rijcke, Dejonghe (2008) nicely reproduce the break at $\mathrm{M}_{V} \sim-14$ mag (dark grey line). Truncating the star-formation histories of the models $0,2,4,6$, and 8 Gyrs ago widens the relation considerably in the dSph regime but does not destroy the relation (light grey asterisks). 
tests, we decided to use 30000 gas particles and 15000 DM particles in the simulations. All model galaxies exhibit episodic star formation. This is a direct consequence of star formation in a shallow potential well. Supernovae heat the gas, causing it to expand. The gas density consequently drops below the threshold for star formation. After the lapse of an adiabatic cooling period, the gas is again allowed to cool and contract. Star formation thus appears to be a self-regulating mechanism.

Since substantial amounts of gas remain in and around the model galaxies throughout the duration of the simulations, one expects tidal interactions and ram-pressure stripping in dense environments to have a significant effect on their SFHs. As a rudimentary way of implementing environmental effects, we simply switch off star formation at some instant in the past and let the stellar population age passively until the present, when the model's observable properties are evaluated. This mimics the effects of ram-pressure stripping or "starvation" (Tully \& Trentham 2008). The cluster potential imposes a Roche limit around a galaxy. Gas outside this limit is no longer available for star formation, leaving the galaxy with only a very limited gas supply to form stars.

\section{Discussion and conclusions}

These results are discussed at length in Valcke, De Rijcke, Dejonghe (2008), De Rijcke et al. (submitted), Penny et al. (submitted), and De Rijcke et al. (in prep.).

There is considerable uniformity in the photometric properties of early-type galaxies, from dwarfs to giants. Photometric parameters quantifying the structure and stellar populations of early-type galaxies, such as the half-light radius, $R_{e}$ the central surface brightness $\mu_{0, V}$, the Sérsic exponent $n$, and V-I color all correlate with galaxy luminosity over a range of more than 6 orders of magnitude in luminosity. We have collected photometric data of dSphs/dEs from different environments (galaxy groups and clusters) but find no significant differences between them.

The scaling relations involving the Sérsic parameters, contrary to previous claims, do not keep a constant slope over the whole luminosity range. The Sérsic exponent $n$ varies with luminosity $L$ as $n \propto L^{0.25-0.3}$ for galaxies brighter than $\mathrm{M}_{V} \approx-14$ mag but scatters around a constant value within the range $n \approx 0.5-1.0$ for fainter dSphs. This is in agreement with the fact that the surface brightness profiles of dSphs can be well approximated by King profiles with a concentration in the range $c \approx 3-10$. Central surface brightness increases with luminosity until the formation of the very brightest, cored ellipticals. At $\mathrm{M}_{V} \approx-14 \mathrm{mag}$, the slope of the $\mathrm{M}_{V}-\mu_{0, V}$ changes abruptly. We show that the $\mathrm{M}_{V}$ vs. $\mathrm{V}-\mathrm{I}$ is essentially a metallicity-luminosity relation of old stellar populations, keeping the same slope over the whole luminosity range investigated here.

Clearly, the absolute magnitude $\mathrm{M}_{V} \approx-14 \mathrm{mag}$ is not just an arbitrary divide between dSphs and dEs. The rather abrupt changes in the slopes of some of the photometric scaling relations suggest that below and above this luminosity, different physical processes dominate the evolution of early type galaxies. Basically, the balance between the steepness of the gravitational potential, which drives gas inwards, and supernova explosions, which blow gas outwards, tips over at this crucial luminosity. The near-independence of these scaling relations with respect to environment can be appreciated by investigating models with truncated star-formation histories. Early truncation of star formation can widen the photometric relations, especially in the very low-mass regime, but does not destroy them. These results will be discussed in more detail elsewhere (De Rijcke et al., in prep.). 


\section{References}

Caldwell N., 1999, AJ, 118, 1230

Carraro G., Chiosi C., Girardi L., \& Lia C., 2001, MNRAS, 335, 335

Conselice C. J., 2003, ApJS, 147, 1

Conselice C. J. \& Gallagher, J. S., III, Wyse R. F. G., 2003, AJ, 125, 66

De Rijcke S., Michielsen D., Dejonghe H., Zeilinger W. W., \& Hau G. K. T., 2005, MNRAS, 360,853 (D05)

De Rijcke S., Zeilinger W. W., Hau G. K. T., Prugniel P., \& Dejonghe H., 2007, ApJ, 659, 1172

Graham A. W. \& Guzmán R., 2003, AJ, 126, 1787 (GG03)

Grebel E. K. \& Gallagher J. S. III, Harbeck D., 2003, AJ, 125, 1926

Irwin M. \& Hatzidimitriou D., 1995,

Marcolini A., D’Ercole A., Brighenti F., \& Recchi S., 2006, MNRAS, 371, 64

Matković A. \& Guzmán R., 2005, MNRAS, 362, 289

McConnachie A. W. \& Irwin M. J., 2006, MNRAS, 365, 1263

McConnachie A. W., Arimoto N., \& Irwin M., 2007, MNRAS, 379, 379

Mieske S., Hilker M., Infante L, \& Mendes de Oliviera C., 2007, A\&A, 463, 503

Nagashima M. \& Yoshii Y., 2004, ApJ, 610, 23

Peletier R. F., 1993, A\&A, 271, 51

Penny S. J. \& Conselice C. J., 2008, MNRAS, 383, 247

Penny S. J., Conselice C. J., De Rijcke S., Held E. V., 2008, submitted to MNRAS

Ricotti M. \& Gnedin N. Y., 2005, ApJ, 629, 259

Saviane I., Held E. V., Piotto G., 1996, A\&A, 315, 40

Schlegel D. J., Finkbeiner D. P., Davis M., 1998, ApJ, 500, 525

Sirianni M., Jee M. J., Benítez N., Blakeslee J. P., Martel A. R., Meurer G., Clampin M., De Marchi G., Ford H. C., Gilliland R., Hartig G. F., Illingworth G. D., Mack J., McCann W. J., 2005, PASP, 117, 1049

Smith Castelli A. V., Bassino L. P., Richtler T., Cellone S. A., Aruta C., Infante L., 2008, MNRAS, 386, 2311

Tully R. B. \& Trentham N., 2008, AJ, 135, 1488

Valcke S., De Rijcke S., Dejonghe H., 2008, accepted for publication in MNRAS, astro$\mathrm{ph} / 0807.0397 \mathrm{~V}$

Zucker D. B., Kniazev A. Y., Martínez-Delgado D., Bell E. F., Rix H.-W., et al., 2007, ApJ, 659, L21 